Relations industrielles

Industrial Relations

\title{
Les Comités d'entreprises
}

\section{Raymond Gérin}

Volume 3, numéro 8, avril 1948

URI : https://id.erudit.org/iderudit/1023614ar

DOI : https://doi.org/10.7202/1023614ar

Aller au sommaire du numéro

\section{Éditeur(s)}

Département des relations industrielles de l’Université Laval

\section{ISSN}

0034-379X (imprimé)

1703-8138 (numérique)

Découvrir la revue

Citer cet article

Gérin, R. (1948). Les Comités d'entreprises. Relations industrielles / Industrial Relations, 3(8), 117-119. https://doi.org/10.7202/1023614ar

Tous droits réservés @ Département des relations industrielles de l’Université Laval, 1948
Ce document est protégé par la loi sur le droit d'auteur. L’utilisation des services d'Érudit (y compris la reproduction) est assujettie à sa politique d'utilisation que vous pouvez consulter en ligne.

https://apropos.erudit.org/fr/usagers/politique-dutilisation/ 
conseiller les programmes d'étude aptes à y conduire.

Au Moyen âge, la formation professionnelle relevait des corporations professionnelles. Beaucoup trop réglementée à ce moment, elle devint presque facultative à la fin du XVIIIe siècle et au début du XIXe. L'évolution des méthodes de travail et l'apparition de la machine amenèrent la création d'industries nouvelles, dont les besoins en main-d'oeuvre étaient fort différents de ceux des anciens métiers.

A la fin du XIXe siècle et au début du XXe, la formation professionnelle devint un problème très complexe, et les gouvernements d'un grand nombre de pays nommèrent des commissions pour en rechercher la solution.

On s'inquiète alors du peu d'intérêt que porte la famille professionnelle à la formation des travailleurs, et on note la très petite part qui lui est réservée dans l'administration et le contrôle de la formation professionnelle.

En 1913, la Commission Royale canadienne chargée de trouver une solution recommande que des représentants des employeurs et des travailleurs des industries pour lesquelles les élèves sont préparés soient invités à faire partie des comités et conseils de contrôle des institutions de formation professionnelle.

Elle ajoute que la coopération de ces personnes avec les éducateurs et les professeurs assurera des programmes d'étude et de travaux d'atelier en étroite correspondance avec les besoins de l'industrie.

De plus, cette coopération aidera à rendre le travail de l'école non seulement plus acceptable par les élèvse et les parents, mais aussi en accord constant avec les désirs et l'expérience de ceux qui sont déjà occupés au développement de l'industrie.

En 1945, la Législature de la province de Québec adopte la loi de l'Aide à l'apprentissage, qui facilite la collaboration effective des employeurs, des travailleurs et de l'école professionnelle. Les industries du bâtiment, de la chaussure, de l'imprimerie, de la lithographie, de la coiffure, de l'automobile et de l'horlogerie se sont déjà prévalues de cette loi.

Souhaitons que cette collaboration continue à se manifester, afin que les apprentis et les diplômés de nos écoles puissent entrer dans la vie professionnelle avec plus d'assurance, convaincus que ceux qui les reçoivent se sont activement intéressés à leur formation.

\section{LES COMITÉS D'ENTREPRISES}

Raymond GERIN

\section{Nature des Comités d'entreprises}

Ces comités varient dans leur constitution, d'un pays à l'autre; mais en général, ce sont des organismes constitués par des représentants des ouvriers élus par les employés des divers départements de l'entreprise, d'une part, et par le patron ou ses représentants, d'autre part. Là où il existe cependant une organisation syndicale, les représentants ouvriers sont désignés dans chaque département par mode d'élection ou de nomination directe par le syndicat reconnu. Là où il n'existe pas d'organisation syndicale, les représentants des ouvriers sont choisis dans chaque département de l'usine par mode de bulletin secret. Ces comités siègent d'ordinaire une fois par mois et traitent de toutes les questions qui ont trait à l'entreprise, sauf celles qui concernent les salaires et les griefs.
Buts des Comités d'entreprises

Les buts de ces comités varient également d'une formule à l'autre, mais ils tendent de plus en plus de nos jours à:

1-Associer très largement l'ouvrier à la vie de l'établissement.

2-Assurer une augmentation de rendement.

3-Contribuer à dégager progressivement une élite ouvrière. ${ }^{1}$

\section{Expérience de Comités d'entreprises}

En France, le gouvernement français rendait obligatoire, le 22 février 1945, les comités d'entreprises dans tous les établissements employant cinquante hommes et plus. Leur compétence por-

(1) Cité dans Les Comités d'Entreprises, Philippe Bayard, p. 95. 
tait: a) sur le plan social, par la gestion mutualiste des oeuvres d'entreprises; b) sur le plan technique, par voie d'avis et suggestions obligatoires de part et d'autre en vue d'accroître la production et d'améliorer le rendement; c) sur lt plan administratif, par voie d'avis et suggestions relativement à la gestion et à la marche générale de l'entreprise, par voie de contrôle du bilan et par voie de participation au conseil d'administration.

En Angleterre, les comités mixtes de production, sous l'impulsion du gouvernement anglais, s'étendent dès 1940 à toutes les entreprises comptant plus cent soixante salariés, grâce à une franche collaboration entre les employeurs et les employés, groupés en des associations puissantes: on assiste à l'établissement de comités faisant l'objet d'accords établis par les parties et sanctionnés par le gouvernement.

Aux Etats-Unis, la deuxième guerre mondiale entraîna un développement énorme de ces comités mixtes de production; mais le gouvernement se contenta de stimuler leur mise sur pied par le truchement d'un organisme de documentation et de propagande.

Au Canada, les comités mixtes de production furent l'objet d'une entente intervenue en 1943 entre les principaux syndicats ouvriers et patronaux, sous l'égide du ministère canadien du Travail. Tous ces comités mixtes de production ayant pour but unique d'accroître l'efficacité de la production, de faciliter une entente mutuelle plus complète et d'orienter toutes les compétences, toutes les connaissances et toutes les initiatives disponibles vers la tâche commune de la victoire . $^{2}$

A côté des comités mixtes de production, l'Association Professionnelle des Industriels mit de l'avant une formule de comité de coopération industrielle qui visait à utiliser les meilleurs éléments des formules françaises et anglaises, en repoussant le caractère obligatoire de l'un et matérialiste de l'autre au bénéfice d'une formule plus humaine, plus chrétienne.

\section{Attitude des parties}

En France, les patrons s'opposèrent, mais sans succès, au caractère obligatoire des comités d'entreprises. Quant aux syndicats, ils ne s'en sont pas montrés dans la suite toujours satisfaits. $\mathrm{Si}$, sur le plan social et technique, les oppositions furent moins vives, sur le plan administratif, ni patrons, ni syndicats n'ont eu lieu d'être satisfaits.

(2) Manuel destiné aux Comités mixtes de production, Ottawa, p. 13.
En Angleterre, deux facteurs favorisèrent une attitude bienveillante des parties intéressées: le caractère non obligatoire des comités d'entreprises et une collaboration déjà vieille entre des organisations syndicales puissantes d'employeurs et d'employés.

Aux Etats-Unis, et pour les mêmes raisons qu'en Angleterre, patrons et syndicats acceptèrent les comités mixtes de production comme une formule pratique d'accélérer la production dans l'état d'urgence qu'avait créé la guerre.

Au Canada, à cause d'un développement syndical ouvrier moins puissant qu'en Angleterre et qu'aux Etats-Unis, on a appréhendé l'introduction des comités mixtes de production. Toutefois, les objections qu'on a formulées contre eux ont consisté en ce que l'on a considéré trop le facteur production et pas assez le caractère humain. C'est à cette objection qu'a voulu répondre l'Association Professionnelle des Industriels en créant son comité de collaboration industrielle.

\section{Résultats}

L'expérience française est trop récente pour qu'on puisse tirer des conclusions absolues à son sujet; mais, « les cas de réussite se constatent là où le patron avait déjà créé par des efforts personnels et opiniâtres un réel climat de collaboration, là où l'on n'a pas abordé la constitution du comité d'entreprise avec un esprit revendicatif du côté des travailleurs et un esprit restrictif du côté patronal ». ${ }^{3}$

En Angleterre, une enquête effectuée auprès des employeurs des industries mécaniques démontre que plus de $75 \%$ des entreprises ont obtenu un résultat satisfaisant des comités mixtes de production.

Aux Etats-Unis, une enquête semblable a démontré que $63 \%$ des maisons enquêtées manifestèrent l'intention de conserver leur comité mixte de production.

Et au Canada, après un certain déclin, le ministère fédéral du Travail donne une augmentation de 54 nouveaux comités, ce qui porte le total à 543 pour tout le Canada au cours des derniers six mois de 1947.

Quant au comité de coopération industrielle de l'Association Professionnelle des Industriels, nous avons personnellement noté des résultats non seulement au point de vue productif mais surtout au point de vue climat d'atelier, vérifié par l'éclo-

(3) Les Comités d'Entreprises en France, une enquête de la F.E.P.A.C., p. 6. 
sion de réalisations sociales d'entreprise de valeur indiscutable.

\section{Conditions de succès}

Mais le succès n'a été obtenu et ne sera obtenu dans les comités d'entreprises que par un sens de compréhension du côté patronal et un sens de responsabilité du côté ouvrier; mais surtout par l'intérêt personnel qu'y mettra le patron, en lui donnant autant d'importance qu'à la partie administrative de son entreprise. Mais avant tout, il faudra viser à une réforme intérieure des individus, gage de la rénovation sociale elle-même.

\section{CLAUSE DE RENOUVELLEMENT DANS LES CONVENTIONS COLLECTIVES}

\section{Georges-Michel GIROUX}

L'Etat, par la Loi des relations ouvrières, a réglementé la durée des conventions collectives et le mode de leur renouvellement. Citons la disposition :

《15. Une convention collective ne peut être conclue pour plus d'un an, mais il peut être convenu qu'elle se renouvellera automatiquement pour une telle période et ainsi de suite au défaut d'une des parties de donner un avis écrit à l'autre partie dans un délai qui ne doit pas être de plus de soixante ni de moins de trente jours avant l'expiration de chaque période. $\gg$

Quelle est la portée juridique de la disposition? C"est précisément l'objet de la présente étude.

Au préalable, cependant, il conviendrait de rappeler la situation avant la promulgation de cette loi, et de mettre en lumière les principes qui ont déterminé l'orientation nouvelle.

En vertu de la Loi des syndicats professionnels, la convention collective de travail devint une entité juridique; on y définit sa nature et on $y$ détermina l'étendue des obligations des personnes qu'elle liait ou assujettissait. Quelques-unes des règles du Code de travail français (a. $31 \&$ s.) furent incorporées dans cette législation, mais les principaux articles donnant à cette institution sa physionomie propre, furent ignorés.

Seule la convention souscrite par un syndicat constitué en corporation eut droit de cité; celle conclue par une union dépourvue de personnalité juridique constituait un \&gentlemen's agreement », i.e. un arrangement non avenu en droit civil.

La loi laissait les parties libres d'en déterminer la durée. Notre législateur, contrairement au Code français, n'a pas précisé, que la conven- tion à terme fixe ne pouvait excéder cinq ans (a. $31 \mathrm{~g})$, que la convention à terme fixe, venue à expiration, se continuait comme convention à durée indéterminée (a. $31 \mathrm{~h}$ ), et que cette dernière cessait sur préavis d'un mois (a. 31m), de s'appliquer à toute personne voulant s'en dégager.

D'après les principes du droit, la durée des conventions collectives avant l'entrée en vigueur du chapitre 162 -A, était assujettie aux règles suivantes :

a) la convention conclue pour une entreprise déterminée ou un terme fixe, sans clause de renouvellement, était automatiquement périmée après que l'entreprise fut achevée ou que le terme fut déchu;

b) la convention conclue sans durée déterminée, à moins de stipulation spéciale, était automatiquement périmée au gré d'une personne qui manifestait sa volonté de s'en dégager; on ne pourait, dans l'expèce, appliquer les règles exceptionnelles de délais-congé de location de maison ou de services.

Mais les parties généralement fixaient, comme dans le cas de location de maison, un terme fixe, ordinairement un an, et prévoyaient le renouvellement de la convention pour un même terme au défaut d'une des parties de s'en dégager par préavis donné dans le délai prévu. Il s'agissait donc de conventions pour un terme précis, se renouvellant de plein droit pour la même durée au défaut d'avis de dégagement.

$\mathrm{La}$ règle à rappeler, c'est que les stipulations de la convention collective sur le terme, sa prorogation, le dégagement d'une partie et le préavis de dégagement, étaient du domaine absolu des parties, le législateur n'ayant pas formulé de règles sur cette matière. 